\title{
Waleed Brinjikji, Timo Krings: Imaging in Neurovascular Disease. A Case-Based Approach
}

\author{
Thieme Publishers, New York/Stuttgart, 2020. ISBN: 9781684200535
}

\author{
Luigi Mansi ${ }^{1}$
}

Published online: 15 April 2020

(C) Springer-Verlag GmbH Germany, part of Springer Nature 2020

Neurovascular imaging has been completely revolutionized in the last decades because of a sequence of new acquisitions started from a time when interventional angiography was alone as a diagnostic and therapeutic option. The advent of computerized imaging created a differentiation between different procedures, with a diagnostic role for invasive angiography remained in conditions in which both approaches have to be associated. At present, without the need of an intraarterial injection, it is possible to acquire very effective angiographic information with ultrasounds (US), computed tomography (CT), and magnetic resonance (MR). Of particular relevance is the contribution allowed by MR, also because of the possibility to add a pathophysiological information, achievable through functional MR (fMRI), to morphostructural and angiographic data.

This publication has been authored by Waleed Brinjikji, an associate professor of radiology and neurosurgery at the Mayo Clinic in Rochester, and Timo Krings, a professor of radiology and surgery at the University of Toronto. Both of them are experts either in neuroimaging or in interventional techniques, with a particular experience in neurovascular diseases.

The paperback of 240 pages in large format is almost an Atlas for the presence of 793 high-quality illustrations. It includes complimentary access to a digital copy on https:// medone.thieme.com.

Organized following a case-based approach, the volume is structured in the following 11 chapters: (1) Acute Ischemic Stroke; (2) Cervical Vascular Disease; (3) Intracranial Steno-
Occlusive Disease; (4) Intracranial Aneurysms; (5) Cerebral Arteriovenous Malformations (AVMs) and Dural Arteriovenous Fistulas (dAVFs); (6) Pediatric Neurovascular Disease; (7) Venous Occlusive Diseases; (8) Angiographically Occult Vascular Lesions; (9) Small Vessel Disease; (10) Intracranial Hemorrhage; (11) Spinal Vascular Disease. The casuistry is mainly based on the most frequent situations, but also rare conditions are included.

Each chapter has a standard organization, starting from a concise clinical introduction to disease, presented through multiple case images. For each pathological condition, the authors report key facts to explain the different roles of all the neurovascular imaging procedures in the disease management. To better individuate relevant findings, insightful clinical pearls and potential pitfalls are also introduced. In this way, it is possible to frame at best each neurovascular disease, either in the diagnostic or in the therapeutic scenario. An updated bibliography creates conditions for a further deepening of specific issues.

A quality of the book is that it provides knowledge to learn the role of vascular imaging not only in diagnosis, prognostic stratification, and treatment decision-making, but also in understanding pathophysiology of neurovascular diseases.

For the reason reported above, I suggest this book to students and professionals involved in Neurosciences, with main reference to neuroradiologists, neurosurgeons, and neurologists.
Luigi Mansi

mansi.luigi@libero.it

1 Interuniversity Research Center for Sustainability (CIRPS),

Naples, Italy 